\title{
Structural and phylogenetic analysis of Pto-type disease resistance gene candidates in Hevea brasiliensis
}

\author{
W. Zhai ${ }^{1,2}$, Y. Zhao', L.X. Zhang ${ }^{1}$ and X.J. Li ${ }^{3}$ \\ ${ }^{1}$ College of Traditional Chinese Medicine, Jilin Agricultural University, \\ Changchun, China \\ ${ }^{2}$ Tropical Crops Genetic Resources Institute of Chinese Academy of Tropical \\ Agricultural Sciences, Danzhou, Hainan, China \\ ${ }^{3}$ Kangmei Xinkaihe (Jilin) Pharmaceutical Co., Ltd., Ji'an, China
}

Corresponding author: L.X. Zhang

E-mail: zlx863@163.com

Genet. Mol. Res. 13 (2): 4348-4360 (2014)

Received January 18, 2013

Accepted August 20, 2013

Published June 10, 2014

DOI http://dx.doi.org/10.4238/2014.June.10.2

\begin{abstract}
The tomato Pto gene encodes a serine/threonine kinase (STK) whose molecular characterization has provided valuable insights into the disease resistance mechanism of tomato. Therefore, Pto is considered as a promising candidate for engineering broad-spectrum pathogen resistance in this crop. In this study, a pair of degenerate primers based on conserved subdomains of plant STKs similar to the tomato Pto protein was used to amplify similar sequences in a hevea cultivar (Hevea brasiliensis Muell. Arg). A fragment of $550 \mathrm{bp}$ was amplified, cloned, and sequenced. The sequence analysis of several clones revealed 12 distinct sequences highly similar to STKs. Based on their significant similarity with the tomato Pto protein (BLASTX $E$ value $<3 \mathrm{e}-53$ ), seven sequences were classified as Pto resistance gene candidates (Pto-RGCs). Multiple sequence alignment of the hevea Pto-RGC products revealed that these sequences contain several conserved subdomains present in most STKs, as well as several conserved residues that are crucial for Pto function. Moreover, phylogenetic analysis
\end{abstract}


showed that the hevea Pto-RGCs clustered with Pto, suggesting a common evolutionary origin with this resistance gene. The Pto-RGCs isolated in this study represent a valuable sequence resource that could assist in the development of disease resistance in hevea.

Key words: Disease resistance gene; Pto; Serine/threonine kinase; Hevea brasiliensis

\section{INTRODUCTION}

Natural rubber is not only an important industrial material but is also an important strategic resource. Hevea cultivars of the Para rubber tree (Hevea brasiliensis Willd. ex A. Juss.) is the only commercial source at present, due to its high rubber content and quality (Backhaus, 1985). Natural rubber production is currently threatened by a wide spectrum of pathogens, including viruses, bacteria, fungi, and nematodes. They reduce yield, affect quality, and debilitate or kill the host plant (Jacob et al., 1989; Lespinasse et al., 2000). Currently, the ascomycete fungus Microcyclus ulei (P.Henn.) v. Arx (South American leaf blight, SALB), which originates from South America, has become the most important disease of the region, even resulting in destruction of some rubber plantations, and there is no indication of the existence of an effective chemical control (Alencar et al., 1975; Albuquerque et al., 1987). Genetic resistance is the most suitable strategy to control these pathogens in the field, and there are sources of resistance in germplasm of wild clones of both species. However, breeding for pathogen resistance in hevea is limited by the long life cycle, triploidy, and sterility characterizing most commercial cultivars. Molecular biotechnology techniques have the potential to overcome these constraints by transferring single or even multiple disease resistance (R) genes into the genome of susceptible hevea cultivars using either biolistics (Narayanan and Kavitha, 2011) or Agrobacterium-mediated transformation. Although transformation technologies have been developed for hevea in various laboratories around the world, no hevea R gene has been isolated to date (Huang et al., 2010).

The tomato Pto gene is one of the best-characterized and most intensively studied R genes (Pedley and Martin, 2003). Pto confers hypersensitive response (HR)-mediated resistance against strains of Pseudomonas syringae pv. tomato that express the avirulence proteins AvrPto or Avr-PtoB (Martin et al., 1993; Kim et al., 2002). Overexpression of Pto in tomato under the control of the strong cauliflower mosaic virus (CaMV) $35 \mathrm{~S}$ promoter has been shown to activate defense responses in the absence of pathogen inoculation. Pto-overexpressing plants show resistance not only to $P$. syringae pv. tomato but also to Xanthomonas campestris pv. vesicatoria and to the fungal pathogen Cladosporium fulvum (Tang et al., 1999). Based on bioinformatics analysis, Pto encodes a cytoplasmic serine/ threonine protein kinase (STK), which contains several conserved subdomains. Southern hybridization using the tomato Pto gene as a probe revealed the presence of Pto-resistance gene candidates (RGCs) in many plant species such as Arabidopsis, bean, soybean, pea, rice, maize, barley, wheat, and sugarcane (Martin et al., 1993). Pto-type disease resistance gene analogs have been successfully amplified from banana, potato, bean, and grapevine among other crops (Vleeshouwers et al., 2001; Vallad et al., 2001; Di Gaspero and Cipriani, 2003; Peraza-Echeverria et al., 2007); however, no molecular characterization of hevea Pto-RGCs has yet been published. Therefore, the objectives of this study were: 1) to obtain Pto-RGCs from hevea using degenerate polymerase chain reaction (PCR) and 2) to determine the structure and phylogenetic relationships of the hevea Pto-RGCs. 


\section{MATERIAL AND METHODS}

\section{Plant material and DNA extraction}

The hevea wild germplasm 'XJ000072' was chosen for PCR amplification of PtoRGC sequences because it shows resistance to a range of hevea pathogens, including the most destructive such as M. ulei and Oidium heveae B.A.Steinm (Narayanan and Kavitha, 2011). The genomic DNA was isolated using the QIAGEN DNeasy Plant Minikit (QIAGEN Inc., Valencia, CA, USA) according to manufacturer instructions.

\section{Degenerate PCR}

A pair of degenerate primers designed by Vallad et al. (2001), forward 5'-TNGGNSANGG NGKNTTYGG-3' and reverse 5'-ACNCCRAANGARTANACRT C-3', was used to amplify the region between the subdomains I and IX of STKs. The degenerate PCR reaction was performed in a 50 $\mu \mathrm{L}$ reaction volume containing $300 \mu \mathrm{M}$ dNTPs, $4 \mu \mathrm{M}$ each degenerate forward and reverse primers, $1 \mathrm{U}$ Taq DNA polymerase (Invitrogen, Carlsbad, CA, USA), $1 \mathrm{X}$ PCR buffer, $1.5 \mathrm{mM} \mathrm{MgCl}$, and approximately $200 \mathrm{ng}$ genomic DNA. PCR conditions were $95^{\circ} \mathrm{C}$ for $3 \mathrm{~min}$, followed by 35 cycles of $95^{\circ} \mathrm{C}$ for $30 \mathrm{~s}, 45^{\circ} \mathrm{C}$ for $30 \mathrm{~s}$, and $72^{\circ} \mathrm{C}$ for $1 \mathrm{~min}$, with an additional 10 min extension at $72^{\circ} \mathrm{C}$.

\section{Cloning and sequencing}

PCR products were visualized on $1 \%$ agarose gel stained with ethidium bromide. A band of the expected size was excised from the gel and purified using the QIAquick Gel Extraction Kit (QIAGEN) according to the manufacturer instructions. Purified PCR products were cloned into the $\mathrm{pGEM}^{\circledR}$-T easy plasmid vector (Invitrogen). Plasmids were transferred by electroporation into Escherichia coli DH5a competent cells. Bacteria were plated onto Luria-Bertani medium containing ampicillin, X-Gal, and isopropyl- $\beta$-D-1-thiogalactopyranoside (IPTG), and recombinant plasmids were chosen by blue/white selection (Sambrook and Russell, 2001). Plasmid DNA was purified by the alkaline lysis method (Sambrook and Russell, 2001) and sequenced using the BigDye terminator sequencing kit version 3.1 (Applied Biosystems) according to manufacturer instructions. The sequencing products were separated with an ABI 3730 automatic sequencer (Applied Biosystems) through the capillary separation service of the Chinese Academy of Agricultural Science. Selected clones were sequenced in both orientations.

\section{Sequence editing, similarity searches, and multiple-sequence alignment}

All sequences were assembled and edited using the programs SEQMAN and EDIT, respectively, of the Lasergene software package version 4.03 (DNASTAR, Madison, WI). The degenerate primer sequences were removed from each sequenced clone so that only the region between the end of subdomain I and the start of subdomain IX of STKs was considered for further analysis. Predicted amino acid sequences were generated using the translate tool of the EDIT program (Lasergene software). Similarity searches were conducted with the BLASTX program (Altschul et al., 1997) through the National Center for Biotechnology Information (NCBI) GenBank database (http://www.ncbi.nlm.nih.gov) using default settings. Percent amino acid identity 
between predicted protein sequences was determined with the MEGALIGN program of Lasergene software using default settings. Determination of conserved amino acids in hevea Pto-RGC sequences was carried out with the programs ClustalX version 2.1 (Larkin et al., 2007) and WebLogo version 3.0 (Crooks et al., 2004) (http://weblogo.berkeley.edu/) using default settings.

\section{Phylogenetic analysis}

Phylogenetic trees were constructed by the neighbor-joining (NJ) method (Saitou and Nei, 1987) using the NJ algorithm implemented in the Molecular Evolutionary Genetics Analysis (MEGA) software version 5.10 (Tamura et al., 2011) with the Poisson correction. Bootstrapping (1000 replicates) was used to evaluate the degree of support for a particular grouping pattern in the phylogenetic tree. Protein sequences belonging to 12 groups of characterized STKs from Arabidopsis thaliana (Hardie, 1999), a phosphoenolpyruvate carboxylase kinase (PEPck) (GenBank accession No. AF162660) from A. thaliana (Hartwell et al., 1999), the tomato Pto protein (GenBank accession No. A49332), and Pto-RGCs from different plant species were retrieved from GenBank for phylogenetic tree construction. The tomato Pto disease resistant protein was used as query in BLASTP (Altschul et al., 1997) searches to retrieve amino acid sequences of Pto-RGCs from GenBank. Only the region between the end of subdomain I and the start of subdomain IX was considered for the phylogenetic tree constructions.

\section{RESULTS}

\section{Identification of Pto resistance gene candidates in hevea}

PCR amplification of hevea genomic DNA using a pair of degenerate primers previously developed by Vallad et al. (2001) generated an expected band of $\sim 550 \mathrm{bp}$. This band was cloned and 50 clones were sequenced. The primer sequences were removed from each sequenced clone for further analysis. Of the 50 sequenced clones (STK-1 to STK-50), 32 presented uninterrupted open reading frames (ORFs), while the other 18 sequences presented multiple stop codons in all reading frames, and as a result they were not further investigated. Similarity searches of the 32 hevea sequences using the BLASTX algorithm (Altschul et al., 1997) against the NCBI non-redundant database revealed significant similarity to known STKs ( $E$ value $<3$ e-53), including the disease resistance protein Pto from tomato. A threshold value of $85 \%$ amino acid identity, which was previously used by Vallad et al. (2001) to classify PtoRGC clones from bean into classes or groups, was used in the present study; therefore, hevea clones with greater than $85 \%$ amino acid identity were considered to be part of the same group.

A total of 12 distinct groups of STK-like sequences were identified, most of which contained redundant or highly similar clones ( $>97 \%$ amino acid identity). Seven groups were designated as Pto-RGCs based on their significant similarity with the tomato Pto disease resistance protein $(E$ value $<3 \mathrm{e}-53)$. The other five groups showed significant similarity to other types of STKs, which are described below. Each group was designated by the name of a single clone representative of the group and used for further analysis. Percent amino acid identity between the predicted amino acid sequence of Pto-RGCs and the corresponding region of the Pto protein ranged from $64.3 \%$ (STK40) to 70.9\% (STK25) (Table 1), whereas amino acid identity among the Pto-RGCs ranged from 70.3\% (STK 33 vs STK 40) to 99.5\% (STK3 vs 
STK12) (Table 1). BLASTX searches also revealed that no Pto-RGCs were highly similar (> $92 \%$ amino acid identity) to hevea sequences present in the GenBank database.

\begin{tabular}{|c|c|c|c|c|c|c|c|c|}
\hline Pto-RGC ${ }^{a}$ & STK3 & STK7 & STK12 & STK25 & STK33 & STK37 & STK40 & Pto \\
\hline STK3 & - & 78.1 & 99.5 & 83.6 & 77.6 & 83.1 & 77.5 & 70.3 \\
\hline STK7 & - & - & 77.6 & 85.8 & 99.5 & 85.8 & 70.9 & 69.2 \\
\hline STK12 & - & - & - & 84.2 & 77.0 & 83.6 & 76.9 & 69.8 \\
\hline STK25 & - & - & - & - & 85.2 & 98.4 & 74.2 & 70.9 \\
\hline STK33 & - & - & - & - & - & 85.2 & 70.3 & 69.8 \\
\hline STK37 & - & - & - & - & - & - & 73.6 & 70.3 \\
\hline STK40 & - & - & - & - & - & - & - & 64.3 \\
\hline Pto & - & - & - & - & - & - & - & - \\
\hline
\end{tabular}

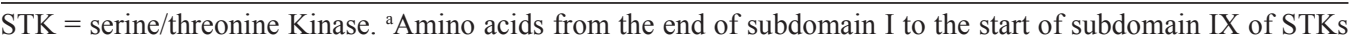
were used for the analysis.

\section{Isolation of other hevea STK-like sequences}

The degenerate primers used in this study were designed from the conserved subdomains I and IX of the STKs Pto, Fen, and Pti1 of tomato, and MHK and APK1 of Arabidopsis (Vallad et al., 2001). Therefore, these primers have the potential to isolate not only Pto-RGCs but also other types of plant STKs. Accordingly, five additional STK-like sequences from hevea were identified in BLASTX searches (Table 2). Four of them (STK8, STK15, STK17, and STK48) showed significant similarity to the receptor-like kinase (RLK) subfamily ( $E$ value $<$ 2e-64), whereas the remaining sequence, STK43, showed significant similarity to a RLK ( $E$ value $=3 \mathrm{e}-123$ ) from Platanus $\mathrm{x}$ acerifolia .

\begin{tabular}{|c|c|c|c|c|c|c|c|}
\hline $\begin{array}{l}\text { STK-like } \\
\text { sequence }\end{array}$ & $\begin{array}{c}\text { GenBank } \\
\text { accession No. }\end{array}$ & Plant & Similar to & $\begin{array}{l}\text { Length of the BLASTX } \\
\text { alignment (in amino acids) }\end{array}$ & $\begin{array}{l}\text { Identity } \\
\%\end{array}$ & $\begin{array}{c}\text { Similarity } \\
\%\end{array}$ & $\begin{array}{l}\text { Expect }(E) \\
\text { value }\end{array}$ \\
\hline STK8 & ABV30720 & Prunus avium & Kinase-like protein & 185 & 74 & 99 & $5 e-94$ \\
\hline STK15 & ABV30718 & Prunus avium & Kinase-like protein & 158 & 77 & 99 & $4 e-96$ \\
\hline STK17 & ABV30718 & Prunus avium & Kinase-like protein & 185 & 77 & 100 & $2 \mathrm{e}-99$ \\
\hline STK43 & AEC04745 & Platanus $\mathrm{x}$ acerifolia & Receptor-like kinase & 187 & 93 & 100 & $3 e-123$ \\
\hline STK48 & ABV30720 & Prunus avium & Kinase-like protein & 184 & 71 & 99 & $8 \mathrm{e}-87$ \\
\hline
\end{tabular}

\section{Multiple sequence alignment and phylogenetic analysis}

A multiple sequence alignment using the ClustalX program was performed with the predicted amino acid sequences of the seven hevea Pto-RGCs and the corresponding region of the tomato Pto protein (Figure 1). The alignment revealed that several features of the Pto protein are highly conserved in hevea Pto-RGCs, such as the STK subdomains internal to the degenerate primer sequences, the presence of the activation domain between subdomains VII and VIII, its internal $\mathrm{P}+1$ loop site, which is responsible for the specific binding of AvrPto (Frederick et al., 1998), and several invariant amino acids distributed throughout the sequences. In addition, three of the four autophosphorylation sites (serine or threonine) in the activation domain of Pto (Sessa et al., 2000) are conserved in the corresponding region of all hevea Pto-RGCs. The alignment also showed that all hevea Pto-RGCs presented a two amino acid deletion (subdomain V) and a three amino acid insertion (subdomain VIa) with 
regard to the Pto protein. We found that the two amino acid deletion was also present in PtoRGCs from other monocot species such as Solanum habrochaites (GenBank accession No. AAK11567), Solanum berthaultii (GenBank accession No. AAK82689), Phaseolus vulgaris (GenBank accession No. AAK52079), Oryza sativa (GenBank accession No. XP476621), and Tritium aestivum (GenBank accession No. AAL51075). This deletion was also present in PtoRGCs from other dicot species, such as A. thaliana (GenBank accession No. NP197789) and Cucumis sativus (GenBank accession no. AAP57674), but was absent in P. vulgaris (GenBank accession No. AF363819). The extent and significance of this polymorphism in both monocot and dicot Pto-RGCs awaits further research. In the case of the three amino acid insertion, it was present in Pto-RGCs from other monocot and dicot species, but was absent in Pto-RGCs from the Solanaceae family (Vleeshouwers et al., 2001).

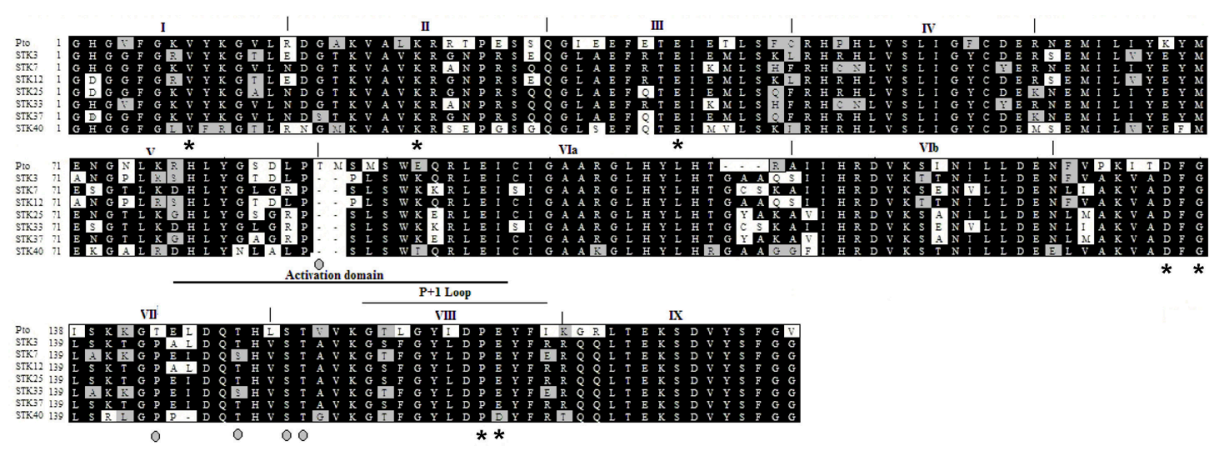

Figure 1. ClustalX alignment of the deduced amino acid sequences of eleven hevea Pto-RGC sequences together with the corresponding region of the Pto protein. The subdomains, activation domain and $\mathrm{P}+1$ loop of protein kinases (Hanks et al., 1988; Hanks and Quinn, 1991; Vallad et al., 2001) are indicated. Conserved amino acids in plant serine/threonine kinases (Hanks and Quinn, 1991) and Pto autophosphorylation sites (Sessa et al., 2000) are indicated with asterisks and gray circles, respectively. Identical amino acids are shaded in black and conservative substitutions are shaded in gray.

In order to highlight the Pto autophosphorylation sites that are conserved in the hevea Pto-RGCs and other critical residues for Pto function located in the activation domain, a sequence Logo was generated with the hevea Pto-RGC products, which is shown in Figure 2. Of the three Pto autophosphorylation sites (Thr195, Ser198, and Thr199) conserved in the hevea Pto-RGCs, Ser198 is required for the AvrPto-Pto-mediated HR (Sessa et al., 2000) and was present in the majority of hevea Pto-RGCs (Figure 2).

The phylogenetic analysis (Figure 3) showed that the seven hevea STK-like sequences identified as Pto-RGCs formed a cluster with the tomato Pto protein, which is supported by a high bootstrap value (99\%). This result supported the designation of the seven hevea STK-like sequences as Pto-RGCs. Regarding the other hevea STK-like sequences, four of them were related to RLKs, confirming the BLASTX results, and the remaining sequence, STK43, formed a highly supported (with bootstrap value 69\%) cluster with a RLKa. (Figure 3). This phylogenetic tree also showed that the protein kinase region used for its construction contained sufficient sequence information to represent clusters defined by analysis with full sequence data of the kinase catalytic domain (Hardie, 1999). Furthermore, phylogenetic analysis of the hevea Pto-RGCs with Pto-RGCs from different plant species (Figure 4) revealed that the hevea Pto- 
RGCs were more closely related to Pto-RGCs from other plant species than to each other. Another interesting finding was that the clades in which the hevea Pto-RGCs were grouped (I, II, and III) also contained Pto-RGCs from different species, suggesting that the origin of this type of sequence may have preceded the divergence of monocots and different plants.

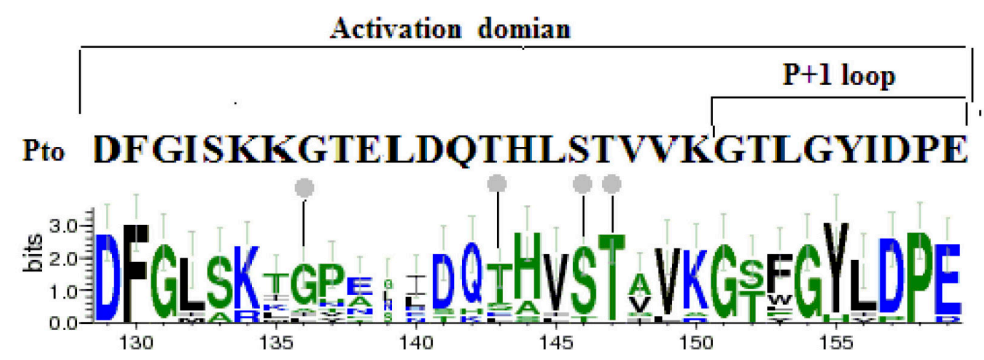

Figure 2. Comparison of the tomato Pto activation domain (30 amino acids in length) with the sequence Logo of the putative activation domain of eleven hevea Pto-RGCs. The Logo sequence consists of stacks of letters, one stack for each position in the sequence. The overall height of each stack indicates the sequence conservation at that position (measured in bits), whereas the height of symbols within the stack reflects the relative frequency of the corresponding amino acid at that position (Crooks et al., 2004). The autophosphorylation sites in the activation domain of Pto (Sessa et al., 2000) are indicated with gray circles.

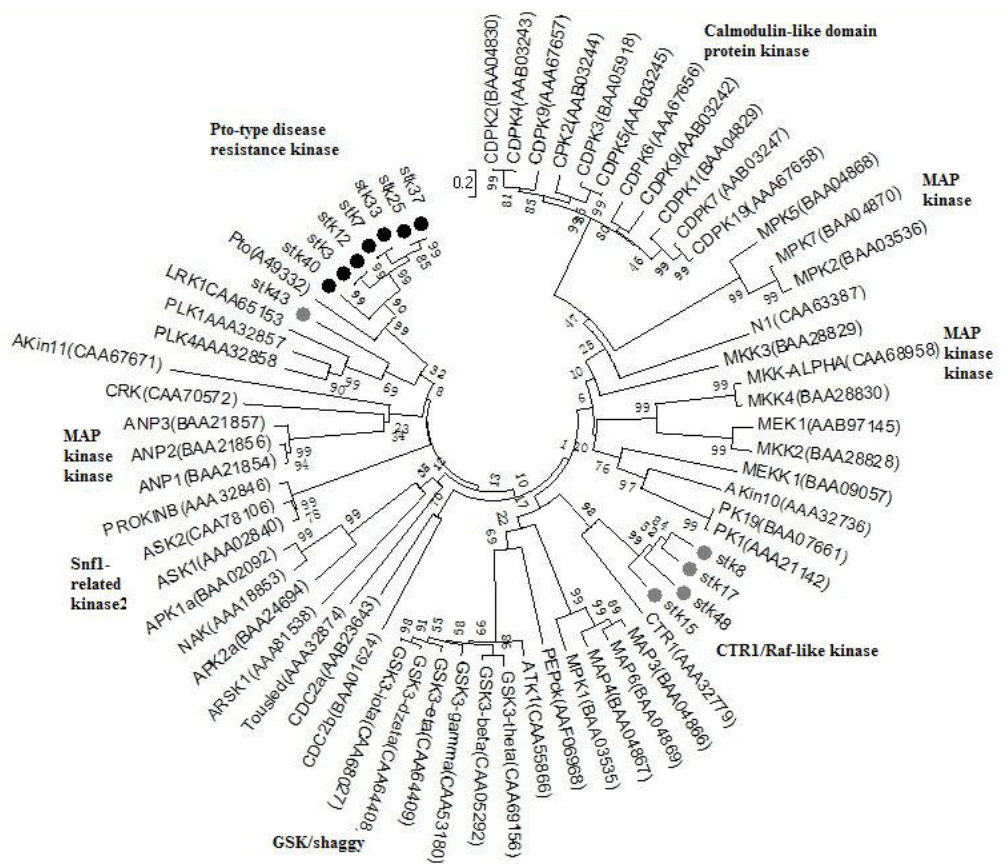

Figure 3. Neighbor-joining phylogenetic tree based on the ClustalX alignment of serine/threonine kinases from Arabidopsis thaliana (Hardie, 1999; Hartwell et al., 1999), tomato Pto disease resistance protein, Pto-RGCs (black circles) and other STK-like sequences (gray circles) from hevea. Names of serine/threonine kinases (STKs) are given for each sequence followed by GenBank accession numbers in parentheses. Amino acids from the end of subdomain I to the start of subdomain IX of STKs were used for the analysis. The numbers above the branches indicate the percentage of 1000 bootstrap replications supporting the particular nodes. 


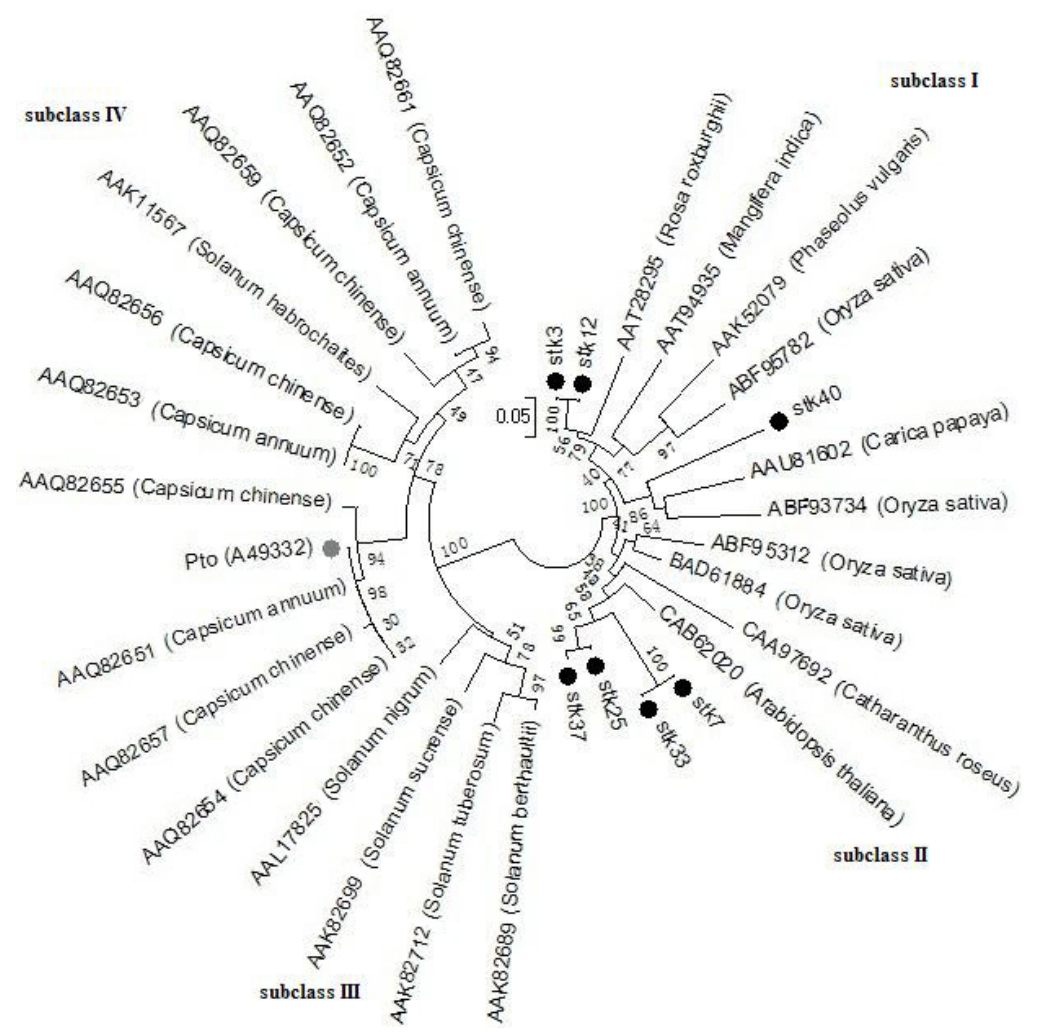

Figure 4. Neighbor-joining phylogenetic tree based on the ClustalX alignment of tomato Pto disease resistance protein, Pto-resistance gene candidates (RGCs) from hevea (black) and other Pto-RGCs from monocot and dicot species. The name of the Pto protein is in bold and GenBank accession Nos. are given for each sequence followed by species name in italics. Amino acids from the end of subdomain I to the start of subdomain IX of serine/ threonine kinases (STKs) were used for the analysis. The numbers above the branches indicate the percentage of 1000 bootstrap replications supporting the particular nodes.

\section{DISCUSSION}

There is evidence that Pto-RGCs are highly conserved in many plant species. Southern hybridization using the tomato Pto gene as a probe revealed the presence of Pto-RGCs in many plant species such as Arabidopsis, bean, soybean, pea, rice, maize, barley, wheat, and sugarcane (Martin et al., 1993). Recent studies have reported the cloning and characterization of Pto-RGCs from potato, bean, and grapevine (Vleeshouwers et al., 2001; Vallad et al., 2001; Di Gaspero and Cipriani, 2003). Furthermore, other Pto-RGC sequences from different plant families have been deposited in GenBank (http://www.ncbi.nlm.nih.gov); however, these sequences have not been characterized. In the present study, a set of Pto-RGC sequences and other STK-like sequences were identified from the hevea cultivar 'XJ000072'. These sequences were isolated by PCR using a pair of degenerate primers previously designed and used by Vallad et al. (2001) to isolate Pto-RGCs from bean. In total, seven distinct Pto-RGC sequences and five other STK-like sequences were identified in the hevea genome. Vallad et al. (2001) reported the identification of a lower number of Pto-RGCs (five distinct sequences 
sharing 56.9 to $63.9 \%$ amino acid identity with Pto) and no further cloning of other STK-like sequences. In the present study, the PCR annealing temperature was lower $\left(45^{\circ} \mathrm{C}\right)$ in comparison to that used in the previous study $\left(60^{\circ} \mathrm{C}\right)$, which may explain the broader diversity of STK-like sequences isolated in hevea. This low PCR annealing temperature could explain the isolation of the receptor-like sequence or the protein-like sequence, which was quite divergent from the rest of the hevea STK-like sequences isolated (Pto-RGCs and RLKs) (Figure 3). Overall, our data demonstrated that the degenerate primers used are capable of amplifying Pto-RGCs and other types of STK-like sequences from a monocot species.

The complete genome sequence of Arabidopsis (genome size of $130 \mathrm{Mbp}$ ) revealed the presence of 15 Pto-RGCs (Arabidopsis Genome Initiative 2000), while a draft of the rice genome sequence (genome size of $420 \mathrm{Mbp}$ ) revealed a similar number of Pto-RGCs with 14 (Goff et al., 2002). These data indicate that the number of Pto-RGCs in these two plant genomes is conserved even though the rice genome is $290 \mathrm{Mbp}$ larger than that of Arabidopsis. This further indicates that the number of Pto-RGCs in plant genomes is small in comparison to the nucleotide binding site-leucine rich repeat (NBS-LRR) class of R genes, which has a large number of divergent genes in the Arabidopsis and rice genomes, with 149 and 480 genes, respectively (Meyers et al., 2003; Zhou et al., 2004). The genome size of hevea is estimated to be $\sim 2100 \mathrm{Mbp}$ (Leitch et al., 1998). Assuming that the number of Pto-RGCs in a plant genome do not increase significantly according to genome size, then it is possible that the number of Pto-RGCs in hevea could be similar to that of Arabidopsis or rice. Hence, it is tempting to speculate that the number of Pto-RGC sequences identified in this study represents a significant proportion of the total number of Pto-RGC sequences in the hevea genome.

All hevea Pto-RGC products displayed conserved serine/threonine kinase sub-domains (Hanks and Quinn, 1991), suggesting that the uncovered genes are likely to encode active kinases. Moreover, most residues of the Pto activation domain involved in pathogen recognition and HR induction (Pedley and Martin, 2003) were found to be highly conserved in hevea Pto-RGCs, suggesting that these residues might play a similar role in hevea. Indeed, the cloning of the full cDNA sequence and protein expression of these hevea Pto-RGCs will allow the possibility to answer some fundamental questions remaining, for example, whether the Pto-RGC encoded proteins are auto-phosphorylated in vitro, and whether substitution of tyrosine by aspartate in the corresponding site of Pto (Tyr207) will lead to an HR-like induction.

Regarding the other STK-like sequences reported in this study, some were similar to RLKs that are known to be involved in pathogen response, for example hevea STK8, STK15, STK17, and STK48 were related to the constitutive triple response 1 (CTR1) group. A member of the CTR1 group is the immediate downstream target of ethylene receptors in A. thaliana, a putative Raf-like MAPK kinase kinase (Kieber et al., 1993). Another interesting example is STK43, which was related to the leucine-rich repeat kinase1 (LRK1) group. LRK1 is an LRR-RLK isolated from A. thaliana, and expression of the gene is induced by abscisic acid, dehydration, high salt, and low temperature (Hong et al., 1997); however, the function of RPK1 remains unclear. The role of other hevea STK-like sequences in disease resistance remains to be determined.

Phylogenetic analyses of Pto and Pto-RGC sequences have suggested that these sequences form a unique group of kinases in plants (Vallad et al., 2001; Vleeshouwers et al., 2001). In agreement with these findings, the hevea Pto-RGCs formed a highly supported group with the Pto disease resistance protein (Figure 3), suggesting that these sequences share a common evolutionary origin with the tomato Pto protein and possibly a similar function in 
disease resistance. Furthermore, phylogenetic analysis of Pto-RGCs from different Solanum species has revealed that Pto ortholog genes are more similar than paralogs, suggesting that the origin of Pto could predate the radiation of Solanum species (Vleeshouwers et al., 2001). This ancient origin of Pto is further supported by the fact that both Pto and a Pto ortholog (LhirPto) are functional in Nicotiana benthamiana (Riely and Martin, 2001). Additional evidence of this ancient origin is the presence of Pto-RGCs in other dicot species as well as monocots that have been recently deposited in GenBank. The phylogenetic analysis shown in Figure 4 supports and extends these previous observations since all hevea Pto-RGCs were grouped in clades that contained Pto-RGCs from both monocot and dicot species, suggesting that the origin of this type of sequence might have predated the divergence of monocot and dicot plants, which took place approximately $200 \pm 40$ million years ago (Wolfe et al., 1989).

The tomato Pto protein is capable of recognizing at least two Avr proteins (AvrPto and AvrPtoB) from P. syringae (Kim et al., 2002). Surprisingly, these two Avr proteins share limited sequence similarity. This dual recognition specificity has also been reported in R proteins of the NBS-LRR class, for example the Rpm1 protein from Arabidopsis confers resistance to P. syringae and recognizes two different avirulence proteins, AvrB and AvrRpm1 (Bisgrove et al., 1994). Another interesting example is the $\mathrm{Mi}-1$ gene from tomato, which confers resistance to both a nematode and an aphid pest (Vos et al., 1998). This dual (and perhaps even multiple) pathogen recognition specificity for a single $\mathrm{R}$ protein may prove to be common in $\mathrm{R}$ genes (Martin et al., 2003) and raises the possibility that Pto may confer resistance to pathogens other than bacteria. Whether the hevea Pto-RGCs are involved in conferring bacterial resistance as in tomato, or are involved in conferring resistance to other types of pathogens, will require functional analysis, which could be carried out with genetic complementation or lossof-function experiments. In the case of genetic complementation, the hevea Pto-RGCs could be used as probes to screen a hevea BIBAC library (Narayanan and Kavitha, 2011) for the isolation of BIBAC clones containing Pto-RGCs. These Pto-RGC-BIBAC clones could be used to transform a hevea disease susceptible cultivar using Agrobacterium tumefaciens (Huang et al., 2010). These experiments would lead to a collection of Pto-RGC-BIBAC transgenic lines ready to be used for disease resistance tests. The BIBAC technology, coupled with Agrobacteriummediated transformation, not only promises to unravel the function of hevea RGCs but also the development of disease resistance in this crop. In the case of the loss-of-function strategy, the hevea Pto-RGC sequences could be used in RNA interference (RNAi) constructs (Waterhouse and Helliwell, 2003) in order to silence their corresponding targets in a resistant genotype. Resistant plants showing disease symptoms after infection with a particular type of pathogen would enable the identification of an R gene. RNAi technology has been recently used to determine the function of genes involved in disease resistance in barley (Douchkov et al., 2005). The hevea Pto-RGCs could also be used to produce molecular markers tightly linked to R genes for genomic mapping and positional cloning. In this respect, several RGCs of the NBS-LRR class have been shown to be quite useful as molecular markers to assist the isolation of functional $\mathrm{R}$ genes through map-based positional cloning (McDowell et al., 1998; Zhao et al., 2005).

The Pto gene is considered to be a promising candidate for engineering broad-spectrum pathogen resistance in tomato since plants overexpressing this gene display resistance to both bacterial and fungal pathogens (Tang et al., 1999). Moreover, expression of Pto mutants, such as ptoThr204Asp or ptoTyr207Asp, can constitutively activate an HR-like response in the absence of $P$. syringae (Rathjen et al., 1999). Expression of these engineered Pto genes 
under the control of a defined inducible promoter has been considered to be another promising strategy to protect crops against pathogens through the HR (Rathjen et al., 1999). Cloning of the full cDNA sequences of the hevea Pto-RGCs will permit assessment of their potential to confer disease resistance using the strategies mentioned above.

In summary, this study uncovered a set of hevea Pto-RGC sequences and provided the first insight into their amino acid sequence structure and evolution. The presence of several conserved amino acids in the hevea Pto-RGCs that are crucial for Pto function, and the fact that these sequences were phylogenetically closely related to Pto, make them a valuable sequence resource for plant-pathogen interaction studies in hevea. These hevea Pto-RGCs could be used to generate not only a collection of BIBAC clones or RNAi constructs for functional analysis, but they might also be useful as molecular markers for genetic mapping. The availability of these sequences will facilitate the cloning of their corresponding full gene sequences, which in turn will allow further genetic and biochemical characterization that may lead to the development of specific or even broad-spectrum pathogen resistance in hevea. Moreover, the other hevea STK-like sequences identified in this study may be used as a research platform for further studies in this crop.

In addition to their potential use for genetic improvement, RGCs also provide opportunities and tools to answer some fundamental questions about disease resistance genes, such as structure, R gene organization, distribution, and evolution (Michelmore and Meyers, 1998; Meyers et al., 1999). The use of PCR with degenerate primers targeting the highly conserved subdomains of STK proteins has also proven to be an efficient method for isolating Pto-RGCs in bean and grapevine (Vallad et al., 2001; Di Gaspero and Cipriani, 2003), indicating that this approach could be used to retrieve this type of gene from other plant species.

\section{REFERENCES}

Albuquerque PEP, Pereira JCR and Santos AF dos (1987). Efficiency of impaction of fungicides by thermal fogging in leaves of Hevea spp. Rev. Theobroma 17: 189-199.

Alencar H, Peixoto E and Ferreira HIS (1975). Controle do mal-das-folhas (Microcyclus ulei) da seringueira na Bahia. II. Relação custo/benefício da aplicação aérea de fungicida, região de Ituberá, 1972-73. Rev. Theobroma 5: 12-20.

Altschul SF, Madden TL, Schaffer AA, Zhang J, et al. (1997). Gapped BLAST and PSI-BLAST: a new generation of protein database search programs. Nucleic Acids Res. 25: 3389-3402.

Backhaus RA (1985). Rubber formation in plants - a mini-review. Israel J. Bot. 34: 283-293.

Bisgrove SR, Simonich MT, Smith NM, Sattler A, et al. (1994). A disease resistance gene in Arabidopsis with specificity for two different pathogen avirulence genes. Plant Cell 6: 927-933.

Crooks GE, Hon G, Chandonia JM and Brenner SE (2004). WebLogo: a sequence logo generator. Genome Res. 14: 1188-1190.

Di Gaspero G and Cipriani G (2003). Nucleotide binding site/leucine-rich repeats, Pto-like and receptor-like kinases related to disease resistance in grapevine. Mol. Genet. Genomics 269: 612-623.

Douchkov D, Nowara D, Zierold U and Schweizer P (2005). A high-throughput gene-silencing system for the functional assessment of defense-related genes in barley epidermal cells. Mol. Plant Microbe Interact. 18: 755-761.

Frederick RD, Thilmony RL, Sessa G and Martin GB (1998). Recognition specificity for the bacterial avirulence protein AvrPto is determined by Thr-204 in the activation loop of the tomato Pto kinase. Mol. Cell 2: 241-245.

Goff SA, Ricke D, Lan TH, Prestring G, et al. (2002). A draft sequence of the rice genome (Oryza sativa L. ssp. japonica). Science 296: 92-100.

Hanks SK and Quinn AM (1991). Protein kinase catalytic domain sequence database: identification of conserved features of primary structure and classification of family members. Methods Enzymol. 200: 38-62.

Hardie DG (1999). Plant protein serine/threonine kinases: classification and functions. Annu. Rev. Plant Physiol. Plant Mol. Biol. 50: 97-131.

Hartwell J, Gill A, Nimmo GA, Wilkins MB, et al. (1999). Phosphoenolpyruvate carboxylase kinase is a novel protein kinase regulated at the level of expression. Plant J. 20:333-342. 
Hong SW, Jon JH, Kwak JM and Nam HG (1997). Identification of a receptor-like protein kinase gene rapidly induced by abscisic acid, dehydration, high salt, and cold treatments in Arabidopsis thaliana. Plant Physiol. 113: 1203-1212.

Huang TD, Li Z and Sun AH (2010). Establishment of Agrobacterium tumefaciens -mediated anther calli transformation system in Hevea brasiliensis. Acta Agron. Sin. 36: 1691-1697.

Jacob JL, Prévôt JC and Roussel D (1989). Hevea Brasiliensis Yield-Limiting Factors, Latex Physiological Parameters, Latex Diagnosis and Clonal Typology. In: Physiology of Rubber Tree Latex (d'Auzac J, Jacob JL and Chrestin H, eds.). CRC Press Inc., Boca Raton, 345-382.

Kieber JJ, Rothenberg M, Roman G, Feldmann KA, et al. (1993). CTR1, a negative regulator of the ethylene response pathway in Arabidopsis, encodes a member of the raf family of protein kinases. Cell 72: 427-441.

Kim YJ, Lin NC and Martin GB (2002). Two distinct Pseudomonas effector proteins interact with the Pto kinase and activate plant immunity. Cell 109: 589-598.

Larkin MA, Blackshields G, Brown NP, Chenna R, et al. (2007). Clustal W and Clustal X version 2.0. Bioinformatics 23: 2947-2948.

Leitch AR, Lim KY and Leitch IJ (1998). Molecular cytogenetic studies in rubber, Hevea brasiliensis Muell. Arg. (Euphorbiaceae). Genome 41: 464-467.

Lespinasse D, Rodier-Goud M and Grivet L (2000). A saturated genetic linkage map of rubber tree (Hevea spp.) based on RFLP, AFLP, microsatellite, and isozyme markers. Theor. Appl. Genet. 100: 127-138.

Martin GB, Brommonschenkel SH, Chunwongse J, Frary A, et al. (1993). Map-based cloning of a protein kinase gene conferring disease resistance in tomato. Science 262: 1432-1436.

Martin GB, Bogdanove AJ and Sessa G (2003). Understanding the function of plant disease resistance proteins. Annu. Rev. Plant Biol. 54: 23-61.

McDowell JM, Dhandaydham M, Long TA, Aarts MG, et al. (1998). Intragenic recombination and diversifying selection contribute to the evolution of downy mildew resistance at the RPP8 locus of Arabidopsis. Plant Cell 10: 1861-1874.

Meyers BC, Dickerman AW, Michelmore RW, Sivaramakrishnan S, et al. (1999). Plant disease resistance genes encode members of an ancient and diverse protein family within the nucleotide-binding superfamily. Plant J. 20: 317-332.

Meyers BC, Kozik A, Griego A, Kuang H, et al. (2003). Genome-wide analysis of NBS-LRR-encoding genes in Arabidopsis. Plant Cell 15: 809-834.

Michelmore RW and Meyers BC (1998). Clusters of resistance genes in plants evolve by divergent selection and a birthand-death process. Genome Res. 8: 1113-1130.

Narayanan C and Kavitha KM (2011). Breeding for Disease Resistance in Hevea spp. - Status, Potential Threats, and Possible Strategies. In: Proceedings of the 4th International Workshop on Genetics of Host-Parasite Interactions in Forestry: Disease and Insect Resistance in Forest Trees, Eugene.

Pedley KF and Martin GB (2003). Molecular basis of Pto-mediated resistance to bacterial speck disease in tomato. Annu. Rev. Phytopathol. 41: 215-243.

Peraza-Echeverria S, James-Kay A, Canto-Canche B and Castillo-Castro E (2007). Structural and phylogenetic analysis of Pto-type disease resistance gene candidates in banana. Mol. Genet. Genomics 278: 443-453.

Rathjen JP, Chang JH, Staskawicz BJ and Michelmore RW (1999). Constitutively active Pto induces a Prf-dependent hypersensitive response in the absence of avrPto. EMBO J. 18: 3232-3240.

Riely BK and Martin GB (2001). Ancient origin of pathogen recognition specificity conferred by the tomato disease resistance gene Pto. Proc. Natl. Acad. Sci. U. S. A. 98: 2059-2064.

Saitou N and Nei M (1987). The neighbor-joining method: a new method for reconstructing phylogenetic trees. Mol. Biol Evol. 4: 406-425.

Sambrook J and Russell DW (2001). Molecular Cloning: A Laboratory Manual. 3rd edn. Cold Spring Harbor Laboratory Press, Cold Spring Harbor.

Sessa G, D'Ascenzo M and Martin GB (2000). Thr38 and Ser198 are Pto autophosphorylation sites required for the AvrPto-Pto-mediated hypersensitive response. EMBO J. 19: 2257-2269.

Tamura K, Peterson D, Peterson N, Stecher G, et al. (2011). MEGA5: molecular evolutionary genetics analysis using maximum likelihood, evolutionary distance, and maximum parsimony methods. Mol. Biol. Evol. 28: 2731-2739.

Tang X, Xie M, Kim YJ, Zhou J, et al. (1999). Overexpression of Pto activates defense responses and confers broad resistance. Plant Cell 11: 15-29.

Vallad G, Rivkin M and Vallejos C (2001). Cloning and homology modelling of a Pto-like protein kinase family of common bean (Phaseolus vulgaris L.). Theor. Appl. Genet. 103: 1046-1058.

Vleeshouwers VG, Martens A, van Dooijeweert W, Colon LT, et al. (2001). Ancient diversification of the Pto kinase family preceded speciation in Solanum. Mol. Plant Microbe Interact. 14: 996-1005.

Vos P, Simons G, Jesse T, Wijbrandi J, et al. (1998). The tomato Mi-1 gene confers resistance to both root-knot nematodes and potato aphids. Nat. Biotechnol. 16: 1365-1369. 
Waterhouse PM and Helliwell CA (2003). Exploring plant genomes by RNA-induced gene silencing. Nat. Rev. Genet. 4: 29-38. Wolfe KH, Gouy M, Yang YW, Sharp PM, et al. (1989). Date of the monocot-dicot divergence estimated from chloroplast DNA sequence data. Proc. Natl. Acad. Sci. U. S. A. 86: 6201-6205.

Zhao B, Lin X, Poland J, Trick H, et al. (2005). A maize resistance gene functions against bacterial streak disease in rice. Proc. Natl. Acad. Sci. U. S. A. 102: 15383-15388.

Zhou T, Wang Y, Chen JQ, Araki H, et al. (2004). Genome-wide identification of NBS genes in japonica rice reveals significant expansion of divergent non-TIR NBS-LRR genes. Mol. Genet. Genomics 271: 402-415. 